\title{
PROCESSING TEST STAND FOR THE FUNDAMENTAL POWER COUPLERS OF THE SPALLATION NEUTRON SOURCE (SNS) SUPERCONDUCTING CAVITIES*
}

\author{
M. Stirbet ${ }^{\dagger}$, I. E. Campisi, G. K. Davis, M. Drury, C. Grenoble, G. Myneni, T. Powers, \\ K. M. Wilson \\ TJNAF, Newport News, VA 23606, USA
}

\begin{abstract}
A test stand has been designed, constructed and commissioned for the processing of the SNS superconducting cavity fundamental power couplers. The cart is designed for insertion into a high-power (1 MW and higher) RF system at $805 \mathrm{MHz}$ and it includes a complete vacuum pumping system, a bakeout control system and, in separate racks, all the controls for the RF conditioning, including interlocks, diagnostics and data acquisition systems.
\end{abstract}

\section{INTRODUCTION}

The fundamental power coupler (FPC) for the SNS Superconducting Cavities [1] must be able to withstand $550 \mathrm{~kW}$ for a pulse duration of $1.3 \mathrm{~ms}$ at $60 \mathrm{pps}$, for a total duty cycle of $8.7 \%$ and an average power of $48 \mathrm{~kW}$. In order to ensure that the FPCs will be able to perform properly in operation, they must be cleaned, assembled, baked and then processed with pulsed RF power at levels about twice the operating power level. A roomtemperature test stand has been designed and built at Jefferson Lab for the characterization, testing and processing of the FPCs before they are assembled onto the superconducting cavities. The test stand allows for simultaneous processing of two FPCs and has the following capabilities: 1) modularity, mobility and clean room compatibility; 2) in situ baking of the FPCs and of the ultrahigh vacuum (UHV) components; 3) monitoring of critical RF and vacuum parameters; 4) diagnostics and control equipment for baking and RF processing; 5) interface with data acquisition and retrieval systems.

The test stand design and its operation will be described in this paper.

\section{MECHANICAL DESIGN}

\subsection{General Description of the FPC Design}

The FPC consists of a tapered 50- $\Omega$ coaxial line. On the vacuum side of the assembly, the outer conductor is double-walled, helium-cooled, copper-plated stainless steel, and the inner conductor is OFE copper. The planar ceramic window assembly has four instrumentation ports on the vacuum side: one for a vacuum gauge, one for an

* Supported by US DOE Contract No. DE-AC05-00OR22725

†stirbet@jlab.org electron pick-up antenna and two sapphire optical ports for infrared and arc detectors. On the air side, the outer extension is made from copper-plated stainless steel and the water-cooled inner extension is copper. The FPC is matched to a rectangular WR975 waveguide via a doorknob/waveguide coaxial transition [2].

To facilitate RF conditioning and ensure safe operation a capacitor for DC bias is provided. Modified Conflat ${ }^{\circledR}$ gaskets are used on the window side to provide good RF contact and, at the same time, a UHV joint [3].

\subsection{Test Cart}

The test stand used for the RF processing of the FPCs includes a robust, mobile aluminum cart, similar to other designs [3], [4], [5]. This houses the vacuum system (1), the connecting waveguide (2) and two SNS FPCs (3), a de-ionized water-compatible cooling manifold (not shown) for the inner conductor extensions and instrumentation needed during baking and/or RF processing. For convenience, the FPCs are tested in an inverted orientation.

The test stand cart has a clean-room compatible module and adjustable feet, which allow the height of the FPCs to be adapted to meet the requirements of the test situation. The test cart and associated components are shown in Figure 1.

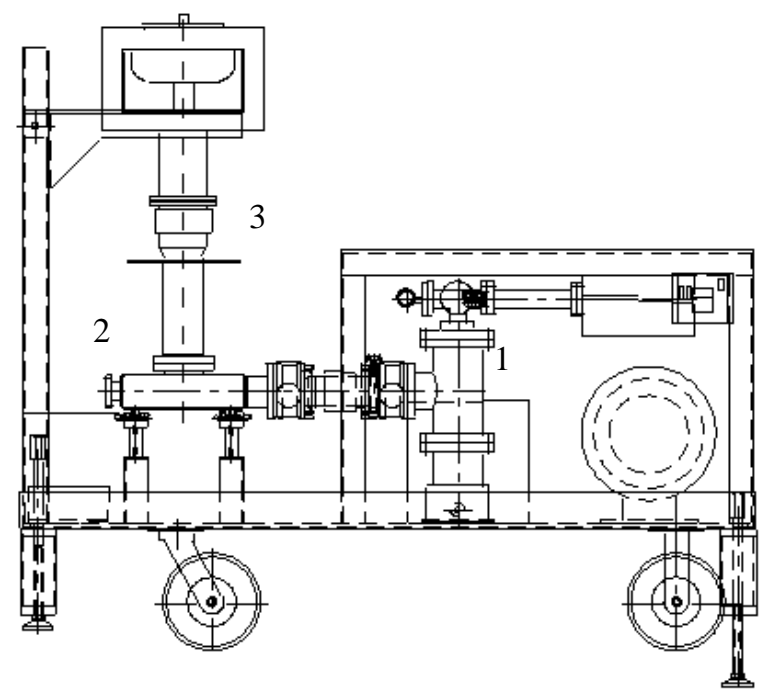

Figure 1: The FPC test cart includes a pumping unit (1), a connecting waveguide (2) and a coupler pair (3). 


\section{CONNECTING WAVEGUIDE}

To allow for simultaneous RF conditioning and power testing of two SNS FPCs under UHV, each pair of FPCs is mounted in a connecting waveguide, operated in transmission mode from one coupler to the other. Since the actual couplers were not available during the design process, the dimensions of the unplated stainless steel connecting waveguide were based on estimated values obtained in numerical simulations [2] and low-power RF measurements using a tapered model of the FPCs and the actual doorknobs in an experimental setup, shown in Figure 2.

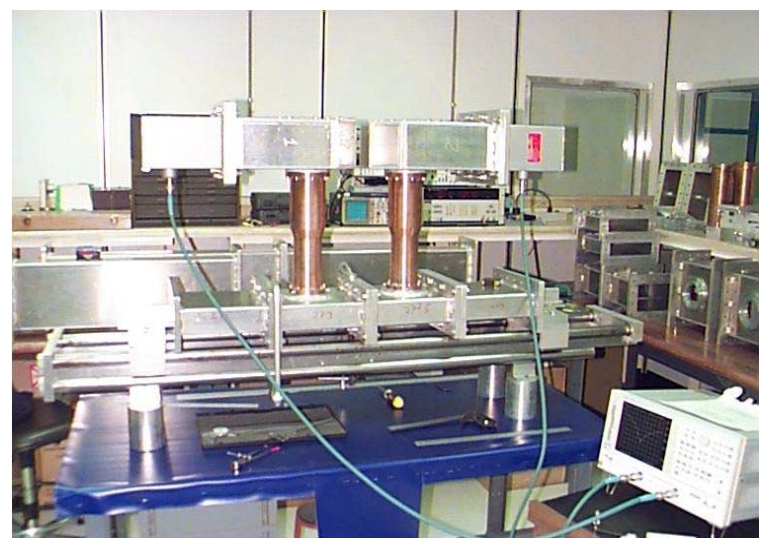

Figure 2: Experimental low-power RF setup for dimensioning the connecting waveguide

The model FPCs were mounted in full- and half-height WR975 waveguides with sliding short circuits at the ends.

However, the outer and inner diameters of the coaxial line were based on an earlier design of the SNS FPC, which might account for some of the differences in the Sparameters values measured on the test stand and on the manufactured connecting waveguide (Table 1).

The differences in S-parameter measurements are also due to contributions from different components in the doorknob transition and the variation in FPC dimensions.

Table 1: S-Parameters at $805 \mathrm{MHz}$ for Connecting HalfHeight Waveguide

\begin{tabular}{|l|l|l|l|l|}
\hline & $\begin{array}{c}\text { S11 } \\
(\mathbf{d B})\end{array}$ & $\begin{array}{c}\text { S21 } \\
(\mathbf{d B})\end{array}$ & $\begin{array}{c}\text { S12 } \\
(\mathbf{d B})\end{array}$ & \multicolumn{1}{|c|}{$\begin{array}{c}\text { S22 } \\
(\mathbf{d B})\end{array}$} \\
\hline Estimated & -34 & -0.94 & -0.91 & -29 \\
\hline Actual & -19 & -0.2 & -0.2 & -19 \\
\hline
\end{tabular}

\section{VACUUM LAYOUT}

Preprocessing and RF conditioning of the couplers is performed under UHV (better than $10^{-9}$ mbar) and materials compatible with this requirement have been considered in designing and manufacturing the test stand.
UHV is maintained using a $260 \mathrm{l} / \mathrm{s}$ turbomolecular pump, which is backed by a Varian ${ }^{\circledR}$ Triscroll dry rough pump. An effective vacuum pump speed of more than $50 \mathrm{l} / \mathrm{s}$ was estimated and subsequently found to be sufficient during baking and RF processing.

Vacuum pressure near the ceramic window is measured with Balzers ${ }^{\circledR}$ inverted magnetron gauges. A compact, full-range $\left(1 \times 10^{-9}\right.$ Torr to 760 Torr $)$ Pfeiffer $^{\circledR}$ gauge, a helium leak standard $\left(\sim 2 \times 10^{-10}\right.$ Torr $\left.1 / \mathrm{s}\right)$ and a Stanford Research System ${ }^{\circledR} 100$ amu residual gas analyzer are mounted on a manifold attached to the connecting waveguide via two all-metal manual valves and a stainless steel bellows.

The vacuum system is compatible with baking at $200^{\circ} \mathrm{C}$. During baking, the external copper surfaces on the window assembly are protected with an argon atmosphere to avoid oxidation. The leak rate (integrated over $\sim 24$ hours) of the test cart with two installed couplers has been measured to be $<2 \times 10^{-10}$ Torr $1 / \mathrm{s}$. After RF processing the couplers and the vacuum system can be backfilled with dry, dust-free nitrogen through a Mykrolis Corporation $^{\circledR}$ nanofilter [6], which is installed on the connecting waveguide.

Two couplers can be stored under nitrogen on the connecting waveguide until they are required for installation on the accelerating cavity.

\section{INSTRUMENTATION AND CONTROLS}

\subsection{Baking System}

The initial baking was performed under vacuum by applying silicon-insulated heating tapes, type $\mathrm{K}$ thermocouples and temperature snap switches. It was controlled using a Programmable Logic Controller (PLC). A LabView-based user interface program was developed for setting the necessary baking parameters and for data acquisition (temperature, vacuum and residual gas analysis). The electronic equipment is located in a standard rack. A small interface chassis placed on the test cart, allows interconnection of heaters, sensors and overtemperature switches (Figure 3).



Figure 3. Test stand with two SNS couplers during baking. 


\subsection{RF Processing}

$\mathrm{RF}$ processing of the main power couplers requires special instrumentation and fast interlocks. These include RF power meters, fast-response vacuum controllers and feedback RF control loop, electrometers, arc detectors and IR detectors. Additionally, flow and temperature of the cooling water in the inner conductor extensions were monitored and interlocked using a PLC. The interlocks were designed to operate independently of computer operation and an interlock fault triggers a PIN diode, which reduces the RF drive signal level by more than $80 \mathrm{~dB}$ in about $1 \mu \mathrm{sec}$.

The instrumentation (shown in Figure 4) is distributed between a PLC, a VXI crate and a number of stand-alone instruments. Data acquisition and controls are accomplished by using a LabView-based program, which also is used to display and log the data acquired by the PLC. The interlocks are implemented in hardware with software monitoring and reset. The VXI instrumentation consists of RF power meters, a multi-channel ADC module, an arbitrary waveform generator, a custom logpower detector module, and a custom interlock and vacuum feedback module. Additionally, a PIN attenuator and PIN switch are mounted in the VXI crate in order to provide hardware interlock control of the RF drive signal.

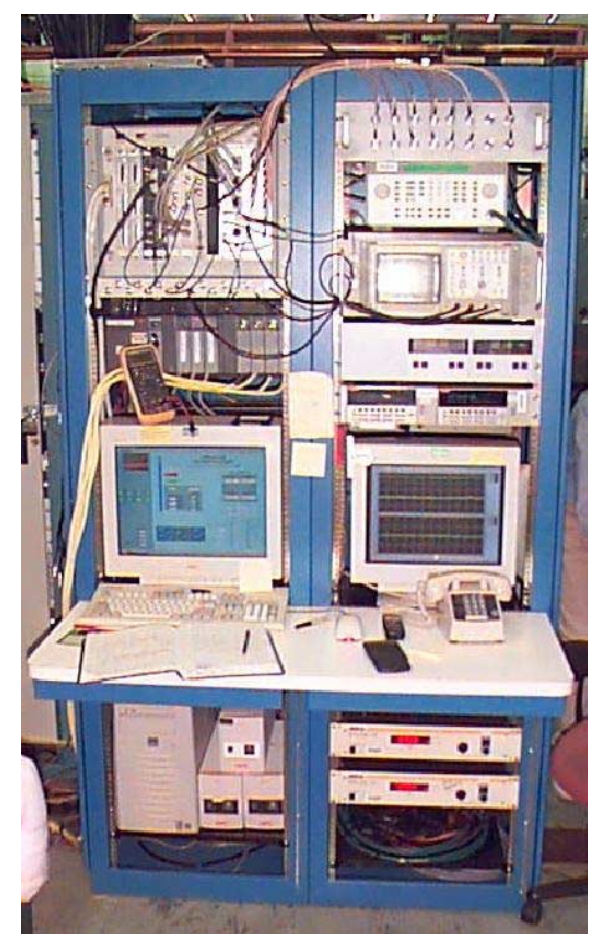

Figure 4: Instrumentation and data acquisition racks for SNS FPC RF processing

\section{INITIAL COMMISSIONING}

Prior to testing at the Los Alamos National Laboratory (LANL), the first two SNS prototype FPCs were assembled at TJNAF on the test cart, baked for 24 hours at $200^{\circ} \mathrm{C}$ and then connected to an $805-\mathrm{MHz}, 20-\mathrm{kW}$ system.

As a system checkout test, $1 \mathrm{~kW}$ of $\mathrm{CW}$ power was transferred though the two FPCs using the control system and protection interlock. This initial commissioning allowed us to check all the aspects of FPC operation at room temperature, from assembly to power control, and to perform a first test of all the safety and operational procedures necessary for RF conditioning and testing of the couplers.

The FPC Test Stand was later transferred to LANL for the initial full power testing. Results of the testing at LANL are discussed elsewhere [7].

\section{CONCLUSIONS}

A room temperature test stand, the associated instrumentation for controls and data acquisition required during preprocessing, RF conditioning and testing the FPCs for SNS has been designed, assembled and commissioned.

Functionality of the adopted design has been demonstrated and solutions for better versions needed in testing the production SNS FPCs identified.

\section{ACKNOWLEDGMENTS}

The authors wish to thank W. Schneider, E. F. Daly, T. Whitlatch, J. Henry, C. Graves, B. Carpenter, K. Macha, L. King, S. Castagnola, C. Apeldoorn, I. Daniels, R. Afanador, T. Elliott, M. Augustine, R. Nichols, D. Gelhaar and W. Sommer for the essential assistance in designing, fabricating, assembling and commissioning the Coupler Test Stand.

\section{REFERENCES}

[1] I. E. Campisi et al., "The Fundamental Power Coupler Prototype for the Spallation Neutron Source (SNS) Superconducting Cavities," PAC 2001, Chicago, IL, June 2001.

[2] Y. Kang et al. "Electromagnetic Simulations and Properties of the Fundamental Power Couplers for the SNS Superconducting Cavities," PAC2001, Chicago, IL, June 2001.

[3] J. Gioia et al., "A Room Temperature Test Bed for Evaluating $700 \mathrm{MHz}$ RF Windows and Power Couplers for the Superconducting Cavities of the APT Linac," PAC 99, New York, NY, April 1999.

[4] D. Proch, personal communication.

[5] E. N. Schmierer et al., "Development of the SCRF Power Coupler for the ATP Accelerator." PAC99, New York, NY, April 1999.

[6] G. Myneni, "Nanofilter for Contamination-Free Backfilling of SRF Structures," JLAB-TN-01-026, Newport News, VA, 2001.

[7] M. Stirbet et al., "Testing Procedures and Results of the Prototype Fundamental Power Coupler for the Spallation Neutron Source," PAC 2001, Chicago, IL, June 2001. 